\title{
1 Role of DNA methylation dynamics in desiccation and salinity 2 stress responses in rice cultivars
}

4 Mohan Singh Rajkumar ${ }^{1}$, Rama Shankar ${ }^{2}$, Rohini Garg ${ }^{3}$ and Mukesh Jain ${ }^{1,2} *$

$6{ }^{1}$ School of Computational \& Integrative Sciences, Jawaharlal Nehru University, New Delhi 7 110067, India,

$8{ }^{2}$ National Institute of Plant Genome Research (NIPGR), Aruna Asaf Ali Marg, New Delhi

9 110067, India and

$10{ }^{3}$ Department of Life Sciences, School of Natural Sciences, Shiv Nadar University, Gautam

11 Buddha Nagar, Uttar Pradesh - 201314, India.

*Correspondence (E-mail: mjain@jnu.ac.in); Telephone: 91-11-26704686

16 MSR: memohansingh@gmail.com

17 RS: $\underline{\text { RamaShankar@hc.msu.edu }}$

18 RG: rohini.garg@snu.edu.in

19 MJ: mjain@jnu.ac.in

21 Running title: DNA methylation dynamics under abiotic stress in rice

23 Number of Figures: 6; Color online only = Figures 1-6

24 Supplementary Data: 1 Table and 4 Figures

25 Word count: 5338

27 Highlight: Bisulphite sequencing revealed single base resolution DNA methylation, and 28 cultivar-specific differential methylation patterns and correlation with gene expression that control desiccation and salinity stress response in the rice cultivars. 


\section{Abstract}

32 DNA methylation is an epigenetic mark that controls gene expression in response to internal and

33 environmental cues. In this study, we sought to understand the role of DNA methylation in 34 response to desiccation and salinity stresses in three rice cultivars (IR64, stress-sensitive; 35 Nagina 22, drought-tolerant and Pokkali, salinity-tolerant) via bisulphite sequencing. We 36 identified DNA methylation patterns in different genomic/genic regions and analysed their 37 correlation with gene expression. Methylation in CG context within gene body and methylation 38 in $\mathrm{CHH}$ context in distal promoter regions were positively correlated with gene expression. 39 However, methylation in other sequence contexts and genic regions was negatively correlated 40 with gene expression. DNA methylation was found to be most dynamic in $\mathrm{CHH}$ context under 41 stress condition(s) in the rice cultivars. The expression profiles of genes involved in de-novo 42 methylation were correlated with methylation dynamics. Hypomethylation in Nagina 22 and 43 hypermethylation in Pokkali in response to desiccation and salinity stress, respectively, were 44 correlated with higher expression of abiotic stress response related genes. Our results suggest an 45 important role of DNA methylation in abiotic stress responses in rice in cultivar-specific 46 manner. This study provides useful resource of DNA methylomes that can be integrated with 47 other data to understand abiotic stress response in rice.

50 Key words: bisulphite sequencing, desiccation stress, DNA methylation, gene expression, rice 51 (Oryza sativa), salinity stress 


\section{Introduction}

54 Abiotic stress is one of the most unpredictable contraventions resulting in crop loss and imposes

55 major concerns in food security. Global warming and adverse environmental conditions result in

56 lower crop yield. Rice is one of the most important cereal crops serving as primary source of

57 dietary carbohydrates in more than half of world population. Drought and salinity stresses affect

58 growth and yield of rice (Bartels et al., 2005; Singh et al., 2018; Peethambaran et al., 2018).

59 Unlike animals, plants are sessile in nature and responses to abiotic stress are associated with

60 activation of gene regulatory networks and pathways involved in stress response (Shinozaki et

61 al., 2007; Zeller et al., 2009). The role of transcription factors and signal transduction

62 components in adaptive stress responses has been revealed (Xiong et al., 2001; Mizoi et al., Zhu

63 et al., 2016; Khan et al., 2018). Epigenetic modifications associated with chromatin re-

64 organization can also play significant role in stress responses (Bruce et al., 2007; Probst et al.,

65 2015; Garg et al., 2015; Neto et al., 2017).

66 DNA methylation is one of most commonly found epigenetic marks. In plants,

67 methylated cytosines are found in three different sequence contexts [CG, CHG and CHH (where

$68 \mathrm{H}=\mathrm{A}, \mathrm{C}$ or $\mathrm{T})$ ]. Maintenance of DNA methylation after each round of DNA replication in

69 daughter stands is mediated by METHYLTRANSERASE 1 (MET1) in CG context and

70 maintenance in CHG context is mediated via CHROMOMETHYLASE 3 (Lindroth et al., 2001;

71 Kankel et al., 2003; Law et al., 2010). De-novo methylation in CHH context is governed by

72 small RNA dependent and independent pathways via DOMAINS REARRANGED

73 METHYLTRANSFERASE 1/2 (DRM1/2) and CHROMOMETHYLASE 2 (CMT2),

74 respectively (Cao et al., 2002; Slothkin et al., 2009; Mosher et al., 2010; Stroud et al., 2014).

75 The possible role of DNA methylation in abiotic stress response has been reported in

76 model and crop plants (Chinnusamy et al., 2009; Lang et al., 2010). Drought induced

77 methylation differences have been identified in sensitive and tolerant cultivars via methylation-

78 sensitive restriction digestion and whole genome bisulphite sequencing in different plants

79 (Wang et al., 2011; Liang et al., 2014; Garg et al., 2015). Interestingly, methylation dynamics

80 under drought stress has been found extensively in $\mathrm{CHH}$ context in both susceptible and tolerant

81 cultivars in Populus trichocarpa (Liang et al., 2014). However, methylation differences between

82 drought-tolerant and susceptible cultivars under control conditions were mostly found in CG

83 context in rice (Garg et al., 2015). Likewise, methylation dynamics under salinity stress have

84 also been investigated in susceptible and tolerant cultivars in different plants using various

85 approaches, and methylation changes under the stress conditions were found to be genotype- 
86 specific (Wang et al., 2014; Al-Lawati et al., 2016; Lu et al., 2017). Interestingly, methylation

87 changes induced in response to abiotic stress condition(s) reversed to its original methylation status after stress recovery (Wang et al., 2011). However, the plants exposed to those abiotic stress(s) were found to adapt more successfully to adverse environmental conditions in subsequent exposures and epigenetic memory was transmitted to subsequent generations (Boyko et al., 2010; Bilichak et al., 2016).

Earlier, we reported DNA methylation patterns and differences between rice cultivars, 93 including stress-sensitive IR64, drought-tolerant Nagina 22 and salinity-tolerant Pokkali (Garg 94 et al., 2015). To further understand the role of DNA methylation in desiccation and salinity 95 stress responses, we sequenced DNA methylomes of rice seedlings exposed to these stresses and 96 compared with that of control conditions. We analyzed the extent of DNA methylation in 97 different sequence contexts at whole genome level. Methylation density and patterns of DNA methylation in protein coding genes and transposable elements (TEs) were revealed. The correlation between DNA methylation density and gene expression levels was analysed under stress conditions in the rice cultivars. We identified differentially methylated regions (DMRs) under stress conditions and their correlation with differential gene expression was revealed. The possible role of DNA methylation in regulation of sets of genes involved in abiotic stress response was also analyzed. Overall, we demonstrated the role of DNA methylation in abiotic stress responses in cultivar-specific manner in rice.

\section{Materials and Methods}

\section{Plant materials and genomic DNA isolation}

108 We analyzed three rice cultivars with contrasting response to abiotic stresses, including tolerant to desiccation (Nagina 22), tolerant to salinity (Pokkali) and sensitive to both stress conditions

110 (IR64). Two-weeks old hydroponically grown rice seedlings were treated with desiccation stress 111 in IR64 and Nagina 22 by keeping them between folds of tissue papers for $3 \mathrm{~h}$. Salinity stress

112 was given by keeping seedlings in $200 \mathrm{mM} \mathrm{NaCl}$ solution for $3 \mathrm{~h}$. The control plants were kept 113 in water for the same duration as described in the previous study (Garg et al., 2015). Stress 114 treated and control seedlings were harvested, snap frozen in liquid nitrogen and stored at $-80{ }^{\circ} \mathrm{C}$

115 till further use. Genomic DNA was isolated using Qiagen DNeasy Minikit (Qiagen) as per 116 manufacturer's instructions. Genomic DNA was quantified using Qubit Fluorimeter (Life 117 Technologies) and purity of DNA was verified by estimating absorbance ratio at 260/280 and 
118 260/230 wavelengths using Spectrophotometer (Nanodrop) and integrity of the DNA was

119 verified by resolving into agarose gel.

\section{Whole genome bisulphite sequencing}

122 To prepare library for bisulphite sequencing, we fragmented the genomic DNA of all the tissue 123 samples to an average size of 100-300 bp via sonication (Covaris, Massachusetts, USA). The 124 genomic fragments were end repaired and TrueSeq-methylated adaptors were ligated to their 125 ends. Adaptor ligated genomic fragments were treated with sodium bisulphite as described in 126 previous study (Garg et al., 2015). Library preparation and sequencing were performed to 127 generate $90 \mathrm{nt}$ long reads in paired-end mode with sufficient sequencing depth (>30x) via 128 HiSeq-2000 platform (Illumina, San Diego, USA).

\section{Read alignment and identification of $\mathbf{m C s}$}

131 The adaptor sequences and low-quality reads were removed from the raw reads using NGSQC 132 Toolkit (v2.3) at default parameters (Patel and Jain, 2012). The clonal reads were filtered out by 133 mapping on the rice genome (MSU v7.0) using Bismark (v0.8) under default parameters in each 134 sample (Krueger and Andrews, 2011). Efficiency of bisulphite conversion was estimated by 135 mapping the high-quality filtered reads on rice chloroplast genome. More than $>99 \%$ of the 136 cytosine(s) in chloroplast genome were converted to thymine(s) indicating very high efficiency 137 of bisulphite conversion in our experiments. The $\mathrm{mCs}$ in rice genome were identified based on 138 the significance of $\leq 0.001 p$-value and sequencing depth of $\geq 5$ reads, as described in previous 139 study (Garg et al., 2015). Methylation level was determined by estimating the percentage of 140 reads giving methylation call at a particular cytosine site to all the reads in the sequencing data 141 covering that site (Garg et al., 2015). Patterns of DNA methylation in rice genome were 142 visualized via circos plot using a window size of $100 \mathrm{~kb}$. Density of DNA methylation in 143 genes/TEs and their $2 \mathrm{~kb}$ flanking regions was calculated using customized perl scripts.

\section{Identification of DMRs}

146 We identified differential methylation under stress conditions as compared to the control 147 condition for each cultivar within each $100 \mathrm{bp}$ bin in the rice genome. The bins covered by $\geq 5$ 148 reads and containing at least 3 cytosine residues were considered for differential methylation 149 analysis. The two bins with same genomic co-ordinates under control and stress conditions were 150 analysed for detection of methylation level difference. Differentially methylated bins showing at 151 least $20 \%$ methylation level difference with $<0.01 q$-value calculated using Fisher's exact test 
152 followed by correction with Sliding Linear Model (SLIM) were determined as described in the

153 previous studies (Garg et al., 2015, Bhatia et al., 2018). Consecutive differentially methylated

154 bins (within a distance of $50 \mathrm{bp}$ ) were merged to identify DMRs and their distribution in

155 different sequence contexts within gene body and $2 \mathrm{~kb}$ flanking regions was analyzed.

Gene ontology (GO) enrichment analysis

158 The enrichment of gene ontology (GO) terms in the sets of DMR associated genes in different 159 cultivars was analysed using BiNGO tool in Cytoscape (v3.7). The significantly enriched GO 160 terms with $q$-value of at least $\leq 0.05$ were identified for each given set of genes.

\section{Integration of DNA methylation and gene expression data}

163 The correlation between DNA methylation and gene expression was determined by plotting 164 methylation density of genes expressed at different levels in each sample. Based on FPKM 165 values, genes expressed at very low ( $<1$ FPKM), low ( $\geq 1-5$ FPKM), moderate $(\geq 5-25$ FPKM) 166 and high (>25 FPKM) levels were categorized. Methylation density in gene body, gene ends and 167 flanking regions was estimated for these sets of genes expressed at varying levels. The 168 correlation between differential methylation and differential gene expression $(\geq 2$ fold-change 169 with $<0.05 q$-value) under desiccation/salinity stress as compared to control condition within 170 same cultivar was analysed by estimating methylation level differences in different sequence contexts within gene body and flanking regions.

\section{Data availability}

174 Bisulphite sequencing and RNA sequencing data reported/used in this study are available under 175 GSE60288 and GSE60287 series accession numbers, respectively, at the Gene Expression 176 Omnibus (GEO) database.

\section{Results and discussion}

\section{Methylome profiling under desiccation and/or salinity stress}

180 To understand the role of DNA methylation in desiccation and salinity stress responses, we 181 profiled DNA methylomes in seedlings of IR64 (sensitive), drought-tolerant (Nagina 22) and 182 salinity-tolerant (Pokkali) rice cultivars after the stress treatment(s) and compared with that of 183 respective control condition. We analysed DNA methylation in IR64 under desiccation and 184 salinity stresses, Nagina 22 under desiccation stress and Pokkali under salinity stress. Bisulphite 
185 sequencing allowed interrogating DNA methylation status of rice genome at single base 186 resolution. We generated about (76-86 million) high-quality 90 bp long paired-end reads with $187>30 x$ sequencing depth for each sample. A total of about 46-55 million uniquely mapped reads 188 covered $\sim 88 \%$ of the rice genome and (81.5-82.8\%) of the total cytosine residues in each 189 sample (Table S1).

190 We estimated percentage of methylcytosines (mCs) with respect to total cytosines in 191 each sequence context that were covered in sequencing for each sample analysed. Interestingly, 192 about half (46.7-49.52\%) of the total cytosines in CG context were found to be methylated in 193 each sample followed by CHG (28.36-30.72\%) and CHH contexts (19.76-24.3\%) (Fig. 1A). 194 Methylation level of mCs in CG context (85.45-90.37\%) was much higher than CHG (66.41195 70.15\%) and $\mathrm{CHH}(39.76-45.69 \%)$ contexts (Fig. 1B; Fig. S1). Previous studies have also 196 shown that methylation levels are generally higher in CG context (Lister et al., 2008; Cokus et 197 al., 2008; Garg et al., 2015; Hossain et al., 2017). It may be due to methylation maintenance in 198 CG and CHG contexts that ensures methylation in the newly formed DNA strands after 199 replication. Another reason for high methylation level in these sequence contexts may be due to 200 KRYPTONITE mediated methylation in CHG context in deep heterochromatic regions 201 associated with H3K9me2 followed by spreading of methylation in CG context (Du et al., 2014; 202 Trejo et al., 2017). In contrast, de-novo methylation in CHH context is dependent on internal 203 and/or environmental cues, which may be the possible reason for low methylation level in $\mathrm{CHH}$ 204 context. Similar methylation levels were found in forward and reverse strands in all the samples analysed (Fig. S2) as reported in previous studies (Jones et al., 2007; Probs et al., 2009; Garg et al., 2015).

To examine differences in patterns of DNA methylation in response to abiotic stress, we compared methylation levels between stress treated and control samples in each cultivar. Interestingly, methylation levels showed most variations in $\mathrm{CHH}$ context followed by $\mathrm{CHG}$ context in response to desiccation and salinity stresses in Nagina 22 and Pokkali, respectively (Fig. 1B). Decreased methylation levels in Nagina 22 under desiccation stress and increased methylation level in Pokkali under salinity stress were observed in both the sequence contexts.

213 However, no obvious methylation level difference was observed in these sequence contexts 214 under desiccation and salinity stress conditions in IR64 with the exception of increased 215 methylation level detected in $\mathrm{CHH}$ context under salinity stress. In CG context, methylation 216 levels were marginally higher under both stress conditions in IR64. In contrast, methylation 217 levels in CG context were marginally lower under desiccation stress in Nagina 22 and under 218 salinity stress in Pokkali. These results showed methylation level differences majorly in $\mathrm{CHH}$ 
219 context under stress conditions, suggesting its important role in determining abiotic stress 220 response.

221 To understand correlation among different samples analysed, we performed clustering 222 among control and stress treated samples of all the rice cultivars based on detected mCs. The 223 control and stress treated sample(s) of the same cultivar clustered together, suggesting that 224 methylome divergence between the cultivars is much higher than the methylome dynamics in 225 response to abiotic stress within a cultivar (Fig. 1C). Further, to examine global methylation 226 patterns, we analyzed the distribution of DNA methylation on the rice chromosomes in all the 227 samples. Interestingly, pericentromeric and centromeric regions harbouring high density of 228 transposable elements (TEs) were found to be extensively methylated in CG and CHG contexts 229 in control and stress treated samples in the rice cultivars. In contrast, higher fraction of mCs in $230 \mathrm{CHH}$ context was detected in gene rich regions under control and stress conditions in all the 231 cultivars, further suggesting the important role of $\mathrm{CHH}$ context DNA methylation in abiotic 232 stress response (Fig. 1D).

\section{DNA methylation in protein coding genes and TEs}

235 We estimated DNA methylation density within the body of protein coding genes and TEs, and their flanking regions in different sequence contexts in all the samples analysed. In general, methylation density in TEs was much higher than protein coding genes in all the sequence contexts. The methylation density at gene ends representing transcription start site (TSS) and transcription termination site (TTS) was much lower than their body and flanking regions in all the sequence contexts. Interestingly, decreased methylation density at TE ends was not observed in CG and CHG contexts, suggesting important role of DNA methylation in TE repression (Fig. 2). Another interesting difference in methylation patterns between genes and TEs was observed in $\mathrm{CHH}$ context. In genes, methylation density at proximal promoter regions (-500 bp) is significantly high in $\mathrm{CHH}$ context, but no such distinct methylation pattern was observed in TEs (Fig. 2).

Next, we analysed differences in methylation density under stress condition(s) as compared to control in the rice cultivars. In Nagina 22, methylation density under desiccation stress was lower as compared to control condition in all the sequence contexts. In contrast, methylation density was higher under desiccation stress in IR64. The difference in methylation density was most evident in the flanking $(-500 \mathrm{bp})$ regions (Fig. 2A). Interestingly, similar differential pattern of DNA methylation profiles between control and desiccation stress treated samples was detected in TEs in both sensitive and tolerant rice cultivars (Fig. 2B; Fig. S3A). 
253 Likewise, methylation density difference between control and salinity stress conditions in IR64 254 and Pokkali cultivars was analysed. Higher methylation density in IR64 and lower methylation 255 density in Pokkali were observed in all the sequence contexts under salinity stress in both 256 protein coding genes and TEs (Fig. 2C, D; Fig. S3B).

257

\section{Influence of DNA methylation on gene expression}

To examine influence of DNA methylation on expression of protein coding genes, we analysed methylation of genes expressed at varying levels under control and stress conditions. The rice genes were classified into sets of genes based on their expression levels, including silent/verylow ( $<1$ FPKM), low ( $\geq 1$ to 5 FPKM), moderate ( $\geq 5$ to 25 FPKM) and high ( $>25$ FPKM) using transcriptome data from our previous study (Shankar et al., 2016). A positive correlation between methylation density in CG context of gene body and expression level was observed as reported in previous studies (Zilberman et al., 2007; Bewick et al., 2017). Interestingly, we observed a positive correlation between DNA methylation in $\mathrm{CHH}$ context in flanking regions ($500 \mathrm{bp}$ ) and gene expression. A positive correlation between DNA methylation in $\mathrm{CHH}$ context in distal promoter regions and TE expression in rice has been reported in previous studies (Zemach et al., 2010a; Zemach et al., 2010b). The mechanism of CHH methylation in promoter regions and enhanced gene expression is largely unknown. In contrast, an antagonistic correlation between DNA methylation in all other genic regions in CG and CHG contexts and gene expression was observed. However, most significant antagonistic correlation of gene expression was found with methylation at transcription start site (TSS) and transcription termination site (TTS) in all the sequence contexts, suggesting that methylation at TSS/TTS may repress gene expression (Fig. 3). These results suggest an important role of DNA methylation in determining expression levels of genes irrespective of different cutivar(s) and/or condition(s).

\section{Differentially methylated regions under abiotic stress in rice cultivars}

280 To study methylation dynamics under desiccation stress in IR64 and Nagina 22 rice cultivars, differentially methylated regions (DMRs) between stress and control conditions were identified 282 for both the cultivars. In total, 2346 and 3013 DMRs representing 2162 and 2744 genes were 283 detected in IR64 and N22, respectively, under desiccation stress. Interestingly, highest number 284 of DMRs was found in CHH context (76.73-77.92\%) followed by CG (15.33-17.22\%) and CHG 285 (4.86-7.93\%) contexts in both the cultivars, suggesting an important role of CHH context DNA 286 methylation in response to desiccation stress (Fig. 4A). Further, we analyzed 
287 hyper/hypomethylation under desiccation stress in different sequence contexts. Interestingly, about $80 \%, 78 \%$ and $71 \%$ of the total DMRs were found to be hypomethylated in CG, CHG and $\mathrm{CHH}$ contexts, respectively, under desiccation stress in Nagina 22. Number of hypermethylated DMRs in CG (59.41\%) and CHH (53.83\%) contexts were marginally higher, while number of 291 hypomethylated DMRs in CHG context (55.26\%) were lower under desiccation stress in IR64 292 (Fig. 4A). Next, we estimated methylation level differences under desiccation stress in both the cultivars. In IR64, fraction of hypermethylated DMRs in CG and CHG contexts was more than hypomethylated DMRs under desiccation stress. In contrast, much higher fraction of DMRs was found to be hypomethylated in all sequence contexts in Nagina 22 (Fig. 4B, C). These results suggest that hypomethylation may be associated with desiccation stress response in Nagina 22. and 3580 DMRs representing 2314 and 3246 genes were detected in IR64 and Pokkali, respectively, under salinity stress (Fig. 4D). Majority of DMRs were detected in CHH context (76.65-77.46) followed by CG (17.92-18.44) and CHG (4.62-4.92) contexts in both the cultivars. In IR64, hypermethylated DMRs in all the sequence contexts; CG (63.56\%), CHG (58.62\%) and $\mathrm{CHH}$ (57.99\%), were more in number under salinity stress. In contrast, hypomethylated DMRs in CG (57.73\%) and CHG (63.64\%) contexts were represented in higher fraction in Pokkali. However, a significant fraction of hypermethylated DMRs in CHH context (80.69\%) was observed under salinity stress in Pokkali cultivar. We analysed methylation level differences under salinity stress in both the cultivars. In IR64, marginally larger fraction of DMRs was associated with hypermethylation in CG and CHG sequence contexts. While, extent of hypermethylation in CHG context was high (Fig. 4E). Interestingly, a very large fraction of DMRs showed hypermethylation in $\mathrm{CHH}$ context in Pokkali, even though higher number of hypomethylated DMRs was detected in CG and CHG contexts (Fig. 4F). This suggests that $\mathrm{CHH}$ context hypermethylation may be involved in salinity stress response in the rice cultivars. genes involved in chromatin remodelling, RNA-dependent DNA methylation (RdDM) pathway and demethylation (Lanciano et al., 2017). Interestingly, lower transcript abundance of OsDRMIa and OsDRMIb methyltransferase genes (involved in methylation) and higher expression level of OSROS1c genes (involved in demethylation) was detected in response to desiccation stress in Nagina 22 as compared to IR64 (Fig. 4G). This suggested that passive methylation and active demethylation in $\mathrm{CHH}$ context might be important for desiccation stress tolerance in Nagina 22. In addition, lower expression of CMT3 under desiccation stress may be the possible reason for hypomethylation in $\mathrm{CHG}$ and $\mathrm{CG}$ contexts in Nagina 22. CMT3 
321 mediated methylation in CHG context and further its role in extending methylation in CG 322 context has been reported ((Du et al., 2014; Trejo et al., 2017). Likewise, we detected higher 323 expression of OsDRM1 (involved in de-novo methylation in $\mathrm{CHH}$ context) and higher 324 expression of genes involved in RdDM pathway, such as $O s D c l 3 b$ and $O s R D R 2$, under salinity 325 stress in Pokkali as compared to IR64. In addition, lower expression of OsDML3a and 326 OsDML3b involved in demethylation was detected. This suggests that RdDM pathway 327 associated hypermethylation in $\mathrm{CHH}$ context and less demethylation may be important for 328 salinity stress tolerance in Pokkali (Fig. 4G).

Our previous analysis showed that methylation differences among the rice cultivars with contrasting responses to drought and salinity stresses were mostly found in CG context (Garg et al., 2015). However, most of methylation changes under stress conditions were detected in $\mathrm{CHH}$ context in this study. Methylation differences in CG context between cultivars and methylation changes in $\mathrm{CHH}$ context within a cultivar under stress have been reported in previous studies too (Liang D et al., 2014; Garg et al., 2015; Lu et al., 2017). This suggests that methylation dynamics in $\mathrm{CHH}$ context guided by de-novo methylation and demethylation may be important during stress response in a cultivar. However, methylation differences detected in CG context between cultivars may be due to diversification of DNA methylomes during selection. Significant methylation in CG context within gene body has been detected in most of the higher plants, but not in lower organisms (Zemach et al., 2010b; Bewick et al., 2016). However, lack of gene body methylation in CG context in an angiosperm (Eutrema salsugineum) was found to be due to loss of CMT3 (Bewick et al., 2016). This suggests that methylation differences

342 detected in CG context between cultivars may be guided by CMT3 in cultivar specific-manner. 343 Interestingly, epigenetic memory was found to be transmitted through DNA methylation in CG 344 context in subsequent generations (Mathieu et al., 2007; Reinders et al., 2009). It is possible that epigenetic memory in subsequent exposures within a generation may be retained through DNA methylation in $\mathrm{CHH}$ context.

Next, we analysed gene ontology (GO) terms represented in hyper/hypomethylated genes under stress conditions. In Nagina 22, GO terms associated with abiotic stress response, including response to desiccation, were enriched in hypomethylated genes. In Pokkali, GO terms related to abiotic stress response, including response to salt stress in the hypermethylated genes (Fig. S4). These results suggest that hypomethylation under desiccation stress in Nagina

35222 and hypermethylation under salinity stress in Pokkali may be important to elicit stress 353 tolerance in these cultivars. 
355 Correlation between differential methylation and differential gene expression under 356 desiccation stress

357 To further understand the role of DNA methylation in response to desiccation stress, we 358 analyzed differential gene expression of DMR associated genes in IR64 and Nagina 22 cultivars 359 under desiccation stress. In IR64, a total of 163 DMR-associated genes exhibited differential 360 expression under desiccation stress (Fig. 5A). Interestingly, $84.1 \%$ of these genes were 361 associated with DMRs in CHH context (Fig. 5C). In Nagina 22, a total of 178 DMR-associated 362 differentially expressed genes were identified and most (87.77\%) of these genes showed 363 differential methylation in $\mathrm{CHH}$ context (Fig. 5B, D). These results suggest that methylation 364 dynamics in $\mathrm{CHH}$ context is involved in desiccation stress response in both the cultivars. Next, 365 we analyzed the DMRs found in CHH context in different gene regions. Interestingly, most (83$36686.5 \%$ ) of the DMRs were located in flanking regions in both the cultivars (Fig. 5B, D). 367 Majority (69.1-71\%) of the CHH context DMR-associated genes showed higher expression in 368 both the cultivars under desiccation stress (Fig. 5B, D). However, a negative correlation of 369 hypomethylation with higher gene expression was observed only in Nagina 22. In IR64, about $37045-50 \%$ of the genes showing hypomethylation in $\mathrm{CHH}$ context in different gene regions exhibited higher expression under desiccation stress, suggesting no obvious correlation between differential methylation and differential gene expression (Fig. 5B). In contrast, about 77-84\% of the genes that showed hypomethylation in $\mathrm{CHH}$ context in different gene regions exhibited enhanced transcript abundance under desiccation stress in Nagina 22 (Fig. 5D).

Next, we analyzed methylation changes under desiccation stress in sets of genes known to be involved in abiotic stress response. In IR64, a total of 18 DMR-associated genes involved in abiotic stress response showed differential expression under desiccation stress. Of these, 12 genes showed enhanced expression and $50 \%$ of them were either hyper/hypomethylated under desiccation stress, suggesting no obvious correlation (Fig. 5C). In contrast, 13 genes representing $81.25 \%$ of the $\mathrm{CHH}$ context DMR-associated genes exhibited hypomethylation and higher gene expression under desiccation stress in Nagina 22 (Fig. 5F). Hypomethylation was found mostly in flanking regions $(92.3 \%)$ for this set of genes. Genes encoding transcription factor(s) and/or those involved in abiotic stress response, including bZIP (LOC_OsO8g36790),

384 zinc finger (LOC_OsO8g38460), AP2 (LOC_Os04g57340), no apical meristem 385 (LOC_OsO2g38130), and homeobox (LOC_OsO1g06560), were found to be correlated with 386 hypomethylation and higher gene expression under desiccation stress in Nagina 22. Gain of 387 function of bZIP (OsbZIP71), zinc finger (OsSAP1), a member of APETELLA 2 (OsAP37) and 388 no apical meristem (NAM) transcription factors conferred tolerance to desiccation stress in rice 
389 (Mukhopadhyay et al., 2004; Oh et al., 2009; Liu et al., 2014; Rahman et al., 2016). A gene

390

391

392

393

394

395

396

397

398

399

400

401

402

403

404

405

406

407

408

409

410

411

412

413

414

415

416

417

418

419

420

421

422

involved in photochemical quenching and dissipation of excess light, zeaxanthin epoxidase gene (LOC_Os04g37619), showed hypomethylation in $\mathrm{CHH}$ context and higher expression. Zeaxanthin epoxidase (ZEP) gene(s) are known to involved in abscisic acid (ABA) synthesis pathway and over-expression of an AtZEP gene elicited tolerance to drought stress in Arabidopsis (Park et al., 2008). Other genes involved in abiotic stress responses, such as cysteine proteinase inhibitor (LOC_OsO1g16430), phospholipase (LOC_OsO9g37100), and cytochrome c oxidase (LOC_Os07g42910) too showed hypomethylation and higher expression under desiccation stress. It has been shown that over-expression of chymotrypsin inhibitor-like 1 (OsCPII) in rice resulted in better survival and higher seed yield under severe drought (Huang et al., 2007). The role of phospholipase in eliciting drought tolerance via modulating calciumsignalling pathway has also been demonstrated in rice (Abreu et al., 2018; Deng et al., 2019). These results suggest that hypomethylation in $\mathrm{CHH}$ context may govern regulation of candidate genes involved in desiccation stress response in Nagina 22.

\section{Correlation between differential methylation and differential gene expression under} salinity stress

To understand the role of DNA methylation in response to salinity stress, we analyzed differential expression of the DMR-associated genes in different sequence contexts and gene regions in IR64 and Pokkali. A total of 39 and 80 DMR-associated differentially expressed genes under salinity stress were detected in IR64 and Pokkali, respectively (Fig. 6A, B). Most (86.8-90\%) of these genes showed differential methylation in $\mathrm{CHH}$ context in both the cultivars (Fig. 6C, D). About (86.8-92\%) of the DMR-associated genes in CHH context showed higher expression under salinity stress in both the cultivars and most of these genes harbored DMRs in their flanking regions $(81.9-86.1 \%)$.

In IR64, $\mathrm{CHH}$ context hypermethylation in gene body (80\%) and downstream regions (66.6\%) was correlated with higher gene expression under salinity stress (Fig. 6B). In Pokkali, correlation of $\mathrm{CHH}$ context hypermethylation and higher gene expression was observed with greater significance in all the gene regions, such as promoter (95\%), gene body $(81.8 \%)$ and downstream (87.5\%) regions (Fig 6E). In addition, the number of genes showing correlation was much higher ( $\sim 5$ times) in Pokkali as compared to IR64. These results suggest that hypermethylation in $\mathrm{CHH}$ context may be associated with salinity stress response in Pokkali. Further, we analyzed correlation of differential methylation with differential gene expression in the sets of genes involved in abiotic stress response. In IR64, four such genes exhibited CHH 
423 context hypermethylation and higher expression under salinity stress (Fig. 6E). In Pokkali, a

424 total of five genes involved in abiotic stress response showed hypermethylation in $\mathrm{CHH}$ context 425 and higher expression under salinity stress (Fig. 6F). A gene encoding transcription factor, 426 homeobox/leucine zipper (LOC_Os04g45810) and other genes involved in abiotic stress 427 response, including dehydrogenase (LOC_OsO6g13720) and glycosyl hydrolase 428 (LOC_Os01g64100) showed hypermethylation in CHH context and higher expression under 429 salinity stress in Pokkali (Fig. 6F). Positive regulation of salinity stress response genes via 430 leucine zipper (bZIP) has been revealed in earlier studies (Xiang et al., 2008; Zhao et al., 2014). 431 Aldehyde dehydrogenase gene(s) play a major role in betaine biosynthesis and were found to be 432 associated with regulation of cellular osmolytes in eliciting salinity tolerance in Arabidopsis and 433 rice (Kishitani et al., 2000; Sunkar et al., 2003). A glycosyl hydrolase gene of rice (OsGH5BG) 434 was also found to be induced in response to salinity stress (Opassiri et al., 2007). These results 435 suggest that candidate genes are activated under salinity stress in the rice cultivars.

436 This is interesting that desiccation stress response in Nagina 22 is mediated via 437 hypomethylation in $\mathrm{CHH}$ context and salinity stress response in Pokkali is mediated via 438 hypermethylation in $\mathrm{CHH}$ context. Previous studies have also demonstrated that 439 hypomethylation associated with higher gene expression under drought/salinity stress response 440 and hypermethylation associated with higher gene expression under salinity stress response 441 (Boyko et al., 2010; Wang et al., 2014; Al-Lawati et al., 2016). These results suggest that abiotic stress response(s) is dependent on cultivar specific manner.

\section{Conclusions}

445 In this study, we showed an important role of DNA methylation in abiotic stress responses in 446 desiccation tolerant (Nagina), salinity tolerant (Pokkali) and sensitive (IR64) rice cultivars.

447 Methylation in $\mathrm{CHH}$ context was found to be most dynamic under desiccation and/or salinity 448 stress conditions in all the rice cultivars. Interestingly, hypomethylation in $\mathrm{CHH}$ context was 449 correlated with higher gene expression under desiccation stress in Nagina 22. In contrast, 450 hypermethylation in $\mathrm{CHH}$ context was correlated with higher gene expression under salinity 451 stress in Pokkali. These results revealed that abiotic stress response is cultivar-specific in rice. 452 Altogether, we provided new insights into role of DNA methylation in response to abiotic stress 453 in rice. 
456 Supplementary data are available at $J X B$ online.

457 Table S1. Summary of bisulphite sequencing data, mapping and methylated cytosines.

458 Fig. S1. Average methylation level in different sequence contexts under control and stress 459 conditions.

460 Fig. S2. Methylation level in forward and reverse strands.

461 Fig. S3. Methylation patterns in CHH context in TEs.

462 Fig. S4. Gene ontology (GO) analysis of differentially methylated genes under abiotic stress 463 conditions.

\section{Acknowledgements}

466 This work was financially supported by the Jawaharlal Nehru University (JNU), New Delhi

471 References

Abreu FRM, Dedicova B, Vianello RP, Lanna AC, de Oliveira JAV, Vieira AF, Morais OP, Mendonça JA, Brondani C. 2018. Overexpression of a phospholipase (OsPLD $\alpha 1$ ) for drought tolerance in upland rice (Oryza sativa L.). Protoplasma 255, 1751-1761.

Al-Lawati A, Al-Bahry S, Victor R, Al-Lawati AH, Yaish MW. 2016. Salt stress alters DNA methylation levels in alfalfa (Medicago spp). Genetics Molecular Research 15, 15018299.

Bartels D, Sunkar R. 2005. Drought and salt tolerance in plants. Crittical Reviews in Plant Science 24, 23-58.

Bewick AJ, Ji L, Niederhuth CE, et al. 2016. On the origin and evolutionary consequences of gene body DNA methylation. Proceedings in National Academy of Sciences 113, 9111-6.

Bewick AJ, Schmitz RJ. 2017. Gene body DNA methylation in plants. Current Opinion in Plant Biology 36, 103-110.

Bhatia H, Khemka N, Jain M, Garg R. 2018. Genome-wide bisulphite-sequencing reveals organ-specific methylation patterns in chickpea. Scientific Reports 8, 9704.

Bilichak A, Kovalchuk I. 2016. Transgenerational response to stress in plants and its application for breeding. Journal of Experimental Botany 67, 2081-92.

Boyko A, Kovalchuk I. 2010. Transgenerational response to stress in Arabidopsis thaliana. Plant Signal Behaviour 5, 995-8. 
Bruce TJA, Matthes MC, Napier JA, Pickett JA. 2007. Stressful "memories" of plants: Evidence and possible mechanisms. Plant Science 173, 603-608.

Cao X, Jacobsen SE. 2002. Locus-specific control of asymmetric and CpNpG methylation by the DRM and CMT3 methyltransferase genes. Proceedings in National Academy of Science 99, 16491-16498.

Chinnusamy V, Zhu JK. 2009. Epigenetic regulation of stress responses in plants. Current Opinion in Plant Biology 12, 133-139.

Cokus SJ, Feng S, Zhang X, Chen Z, Merriman B, Haudenschild CD, Pradhan S, Nelson SF, Pellegrini M, Jacobsen SE. 2008. Shotgun bisulphite sequencing of the Arabidopsis genome reveals DNA methylation patterning. Nature 452, 215-9.

Deng X, Yuan S, Cao H, Lam SM, Shui G, Hong Y, Wang X. 2019. Phosphatidylinositolhydrolyzing phospholipase $\mathrm{C} 4$ modulates rice response to salt and drought. Plant Cell Environment 42, 536-548.

Du J, Johnson LM, Groth M, Feng S, Hale CJ, Li S, Vashisht AA, Wohlschlegel JA, Patel DJ, Jacobsen SE. 2014. Mechanism of DNA methylation-directed histone methylation by KRYPTONITE. Molecular Cell 7, 495-504.

Garg R, Narayana Chevala V, Shankar R, Jain M. 2015. Divergent DNA methylation patterns associated with gene expression in rice cultivars with contrasting drought and salinity stress response. Scientific Reports 5,14922.

Hossain MS, Kawakatsu T, Kim KD, et al. 2017. Divergent cytosine DNA methylation patterns in single-cell soybean root hairs. New Phytologist 214, 808-819.

Huang Y, Xiao B, Xiong L. 2007. Characterization of a stress responsive proteinase inhibitor gene with positive effect in improving drought resistance in rice. Planta 226, 73-85.

Jones PA, Liang G. 2009. Rethinking how DNA methylation patterns are maintained. Nature Reviews Genetics 10, 805-811.

Kankel MW, Ramsey DE, Stokes TL, Flowers SK, Haag JR, Jeddeloh JA, Riddle NC, Verbsky ML, Richards EJ. 2003. Arabidopsis MET1 cytosine methyltransferase mutants. Genetics 163, 1109-1122.

Khan SA, Li MZ, Wang SM, Yin HJ. 2018. Revisiting the role of plant transcription factors in the battle against abiotic stress. Integrative Journal of Molecular Sciences 19, E1634.

Kishitani S, Takanami T, Suzuki M, Oikawa M, Yokoi S, Ishitani M, Alvarez $\square$ Nakase AM, Takabe T, Takabe T. 2000. Compatibility of glycinebetaine in rice plants: evaluation using transgenic rice plants with a gene for peroxisomal betaine aldehyde dehydrogenase from barley. Plant Cell Environment 42, 536-548. 23, 107-114. 
Krueger F. and Andrews SR. 2011. Bismark: a flexible aligner and methylation caller for Bisulfite-Seq applications. Bioinformatics 27, 1571-1572.

Lanciano S, Mirouze M. 2017. DNA methylation in rice and relevance for breeding. Epigenomes 2, 10.

Lang-Mladek C, Popova O, Kiok K, Berlinger M, Rakic B, Aufsatz W, Jonak C, Hauser MT, Luschnig C. 2010. Transgenerational inheritance and resetting of stress-induced loss of epigenetic gene silencing in Arabidopsis. Molecular Plant 3, 594-602.

Law JA, Jacobsen SE. 2010. Establishing, maintaining and modifying DNA methylation patterns in plants and animals. Nature Reviews Genetics 11, 204-220.

Liang D, Zhang Z, Wu H, et al. 2014. Single-base-resolution methylomes of Populus trichocarpa reveal the association between DNA methylation and drought stress. BMC Genetics 18, 297.

Lindroth AM, Cao X, Jackson JP, Zilberman D, McCallum CM, Henikoff S, Jacobsen SE. 2001. Requirement of CHROMOMETHYLASE3 for maintenance of CpXpG methylation. Science 292, 2077-80.

Lister R, O'Malley RC, Tonti-Filippini J, Gregory BD, Berry CC, Millar AH, Ecker JR. 2008. Highly integrated single-base resolution maps of the epigenome in Arabidopsis. Cell 133, 523-36.

Liu C, Mao B, Ou S, Wang W, Liu L, Wu Y, Chu C, Wang X. 2014. OsbZIP71, a bZIP transcription factor, confers salinity and drought tolerance in rice. Plant Molecular Biology 84,19-36.

Lu XK, Shu N, Wang JJ, Chen XG, Wang DL, Wang S, Fan WL, Guo XN, Guo LX, Ye WW. 2017. Genome-wide analysis of salinity-stress induced DNA methylation alterations in cotton (Gossypium hirsutum L.) using the Me-DIP sequencing technology. Genetics Molecular Research 16.

Mathieu O, Reinders J, Caikovski M, Smathajitt C, Paszkowski J. 2007. Transgenerational stability of the Arabidopsis epigenome is coordinated by CG methylation. Cell 130, 851-62.

Mizoi J, Shinozaki K, Yamaguchi-Shinozaki K. 2012. AP2/ERF family transcription factors in plant abiotic stress responses. Biochimica Biophysica Acta 1819, 86-96.

Mosher RA, Melnyk CW. 2010. siRNAs and DNA methylation: seedy epigenetics. Trends in Plant Sciences 15, 204-210.

Mukhopadhyay A, Vij S, Tyagi AK. 2004. Overexpression of a zinc-finger protein gene from rice confers tolerance to cold, dehydration, and salt stress in transgenic tobacco. Proceedings in National Academy of Science 101, 6309-6314. 
Neto JRCF, da Silva MD, Pandolfi V, Crovella S, Benko-Iseppon AM, Kido EA. 2017. Epigenetic signals on plant adaptation: a biotic stress perspective. Current Protein and Peptide Science 18, 352-367.

Oh SJ, Kim YS, Kwon CW, Park HK, Jeong JS, Kim JK. 2009. Overexpression of the transcription factor AP37 in rice improves grain yield under drought conditions. Plant Physiology 150, 1368-79.

Opassiri R, Pomthong B, Akiyama T, Nakphaichit M, Onkoksoong T, Ketudat Cairns M, Ketudat Cairns JR. 2007. A stress-induced rice (Oryza sativa L.) beta-glucosidase represents a new subfamily of glycosyl hydrolase family 5 containing a fascin-like domain. Biochemistry Journal 408, $241-9$.

Park HY, Seok HY, Park BK, Kim SH, Goh CH, Lee BH, Lee CH, Moon YH. 2008. Overexpression of Arabidopsis ZEP enhances tolerance to osmotic stress. Biochemical Biophysical Research Communications 375, 80-5.

Patel RK and Jain M. 2012. NGS QC Toolkit: a toolkit for quality control of next generation sequencing data. PLoS One 7, e30619.

Peethambaran PK, Glenz R, Höninger S, et al. 2018. Salt-inducible expression of OsJAZ8 improves resilience against salt-stress. BMC Plant Biology 18, 311.

Probs AV, Dunleavy E, Almouzni G. 2009. Epigenetic inheritance during the cell cycle. Nature Reviews Molecular Cell Biology 10, 192-206.

Probst AV, Mittelsten SO. 2015. Stress-induced structural changes in plant chromatin. Current Opinion in Plant Biology 27, 8-16.

Rahman H, Ramanathan V, Nallathambi J, Duraialagaraja S, Muthurajan R. 2016. Overexpression of a NAC 67 transcription factor from finger millet (Eleusine coracana L.) confers tolerance against salinity and drought stress in rice. BMC Biotechnology 11,16.

Reinders J, Wulff BB, Mirouze M, Marí-Ordóñez A, Dapp M, Rozhon W, Bucher E, Theiler G, Paszkowski J. 2009. Compromised stability of DNA methylation and transposon immobilization in mosaic Arabidopsis epigenomes. Genes and Development 23, 939-50.

Shankar R, Bhattacharjee A, Jain M. 2016. Transcriptome analysis in different rice cultivars provides novel insights into desiccation and salinity stress responses. Scientific Reports $\mathbf{6}$, 23719.

Shinozaki K, Yamaguchi-Shinozaki K. 2007. Gene networks involved in drought stress response and tolerance. Journal of Experimental Botany 58, 221-227. 
Singh S, Prasad S, Yadav V, Kumar A, Jaiswal B, Adesh Kumar A, Khan NA, Dwivedi DK. 2018. Effect of drought stress on yield and yield components of rice (Oryza sativa L.) genotypes. Integrative Journal of Current Microbiology and Applied Science 7, 2752-2759.

Slotkin RK, Vaughn M, Borges F, Tanurdzić M, Becker JD, Feijó JA, Martienssen RA. 2009. Epigenetic reprogramming and small RNA silencing of transposable elements in pollen. Cell 136, 461-72.

Stroud H, Do T, Du J, Zhong X, Feng S, Johnson L, Patel DJ, Jacobsen SE. 2014. Non-CG methylation patterns shape the epigenetic landscape in Arabidopsis. Nature Structural Molecular Biology 21, 64-72.

Sunkar R, Bartels D, Kirch HH. 2003. Overexpression of a stress-inducible aldehyde dehydrogenase gene from Arabidopsis thaliana in transgenic plants improves stress tolerance.

Trejo-Arellano MS, Mahrez W, Nakamura M, Moreno-Romero J, Nanni P, Köhler C, Hennig L. 2017. H3K23me1 is an evolutionarily conserved histone modification associated with CG DNA methylation in Arabidopsis. Plant Journal 90, 293-303.

Wang M, Qin L, Xie C, Li W, Yuan J, Kong L, Yu W, Xia G, Liu S. 2014. Induced and constitutive DNA methylation in a salinity-tolerant wheat introgression line. Plant Cell Physiology 55, 1354-65.

Wang WS, Pan YJ, Zhao XQ, Dwivedi D, Zhu LH, Ali J, Fu BY, Li ZK. 2011. Droughtinduced site-specific DNA methylation and its association with drought tolerance in rice (Oryza sativa L.). Journal of Experimental Botany 62, 1951-60.

Xiang Y, Tang N, Du H, Ye H, Xiong L. 2008. Characterization of OsbZIP23 as a key player of the basic leucine zipper transcription factor family for conferring abscisic acid sensitivity and salinity and drought tolerance in rice. Plant Journal 35, 452-64.

Xiong L, Zhu JK. 2001. Abiotic stress signal transduction in plants: Molecular and genetic perspectives. Physiologia Plantarum 112, 152-166.

Zeller G, Henz SR, Widmer CK, Sachsenberg T, Rätsch G, Weigel D, Laubinger S. 2009. Stress $\square$ induced changes in the Arabidopsis thaliana transcriptome analyzed using whole $\square$ genome tiling arrays. Plant Journal 58, 1068-1082.

Zemach A, Kim MY, Silva P, Rodrigues JA, Dotson B, Brooks MD, Zilberman D. 2010a. Local DNA hypomethylation activates genes in rice endosperm. Proceedings in National Academy of Sciences 107, 18729-34.

Zemach A, McDaniel E, Silva P, Zilberman D. 2010b. Genome-wide evolutionary analysis of eukaryotic DNA methylation. Science 14, 916-9. 
Zhao Y, Ma Q, Jin X, Peng X, Liu J, Deng L, Yan H, Sheng L, Jiang H, Cheng B. 2014.A novel maize homeodomain-leucine zipper (HD-Zip) I gene, Zmhdz10, positively regulates drought and salt tolerance in both rice and Arabidopsis. Plant Cell Physiology 55, 1142-56.

Zhu JK. 2016. Abiotic stress signaling and responses in plants. Cell 167, 313-324.

Zilberman D, Gehring M, Tran RK, Ballinger T, Henikoff S. 2007. Genome-wide analysis of Arabidopsis thaliana DNA methylation uncovers an interdependence between methylation and transcription. Nature Genetics 39, 61-69. 


\section{Figure legends}

Fig. 1. Methylation patterns in the rice cultivars under desiccation and salinity stresses. (A) Pie chart showing percentage of methylated cytosines in different sequence contexts under control and stress conditions in the three rice cultivars. (B) Methylation levels in different sequence contexts under control and stress conditions are shown in boxplot. (C) Dendrogram showing similarity/divergence of methylation patterns among the rice cultivars under control and stress conditions are shown. (D) Methylation density (number of methylated cytosines per $100 \mathrm{~kb}$ ) in rice genome under control and stress conditions is shown via circos plot. Gene and TE density has also been shown in the two outermost circles. Ct, control; Ds, desiccation stress; Ss, salinity stress; N22, Nagina 22; PK, Pokkali.

Fig. 2. Methylation patterns in genes and transposable elements (TEs) under desiccation and/or salinity stresses in the rice cultivars. (A, B) Methylation density in IR64 and Nagina 22 is shown in genes (A) and TEs (B) in different sequence contexts under control and desiccation stress conditions. (C, D) Methylation density in genes (C) and TEs (D) in IR64 and Pokkali under control and salinity stress conditions is shown. Methylation density indicates number of methylcytosines (mCs) per $100 \mathrm{bp}$.

Fig. 3. Influence of DNA methylation on gene expression. (A-G) Methylation density of genes expressed at different levels [silent/very-low ( $<1$ FPKM), low ( $\geq 1$ to 5 FPKM), moderate ( $\geq 5$ to 25 FPKM) and high (>25 FPKM)] under control [IR64 (A), Nagina 22 (D), Pokkali (F)], desiccation stress [IR64 (B), Nagina 22 (E)] and salinity stress [IR64 (C), Pokkali (G)] conditions are shown. Up, upstream region, GB, gene body; D, downstream region.

Fig. 4. Methylation dynamics in rice cultivars under desiccation and/or salinity stresses. (A) Number of differentially methylated regions (DMRs) in different sequence contexts under desiccation stress in IR64 and Nagina 22 are shown. (B, C) Methylation level differences in different sequence contexts under desiccation stress in IR64 (B) and Nagina 22 (C) are shown via kernel density plots. (D-F) Number of DMRs in different sequence contexts under salinity stress in IR64 and Pokkali are shown (D) and methylation level differences in different sequence contexts under salinity stress in IR64 (E) and Pokkali (F) are shown. (G) Differential expression profiles of genes encoding DNA methyltransferases, DNA glycosylases, and genes involved in RdDM pathway and chromatin remodeling under desiccation stress in IR64 and 
Nagina 22, and under salinity stress in IR64 and Pokkali are shown via heatmap. Scale at the bottom represents $\log _{2}$ fold-change of expression under stress conditions.

Fig. 5. Differential methylation associated with desiccation stress response in rice. $(A, B)$ Number of DMR associated differentially expressed genes under desiccation stress in IR64 (A) and Nagina 22 (B) is given. (C, D) Differential methylation profiles of the up/down-regulated genes in different sequence contexts and gene regions in IR64 (C) and Nagina 22 (D) are shown. (E, F) Differential methylation (CHH context) profiles of up/down-regulated genes involved in abiotic stress response in IR64 (E) and Nagina 22 (F) are shown. The right most heatmaps show differential gene expression profiles under the stress conditions in IR64 (E) and N22 (F). Scales at the bottom represent methylation level difference (blue, hypomethylation; yellow, hypermethylation) and expression $\log _{2}$ fold-change (red, upregulation; green, downregulation) under stress condition.

Fig. 6. Differential methylation associated with salinity stress response in rice. $(A, B)$ Number of DMR associated differentially expressed genes under desiccation stress in IR64 (A) and Pokkali (B) is given. (C, D) Differential methylation profiles of the up/down-regulated genes in different sequence contexts and gene regions in IR64 (C) and Pokkali (D) are shown. (E, F) Differential methylation ( $\mathrm{CHH}$ context) profiles of the up/down-regulated genes involved in abiotic stress response in IR64 (E) and Pokkali (F) is shown. The right most heatmaps in show differential gene expression profiles under the stress conditions in IR64 (E) and Pokkali (F). Scales at the bottom represent methylation level difference (blue, hypomethylation; yellow, hypermethylation) and expression $\log _{2}$ fold-change (red, upregulation; green, downregulation) under stress condition. 
A

$\square$ cG $\square$ CHG $\square$ CHH

D
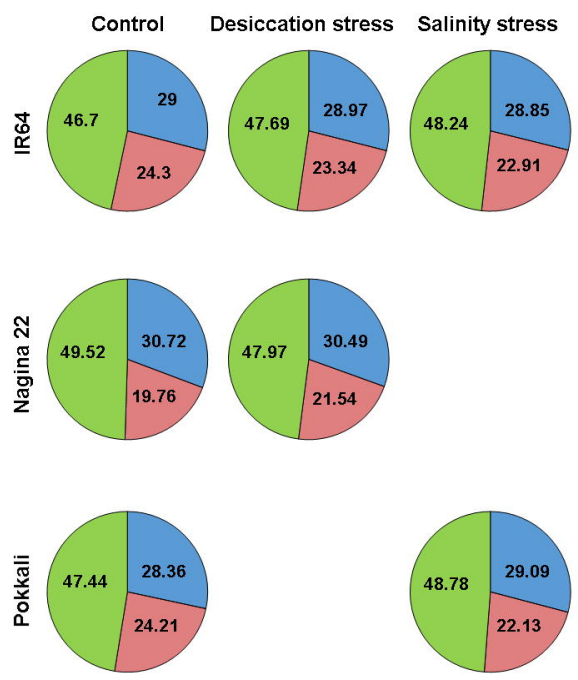

B

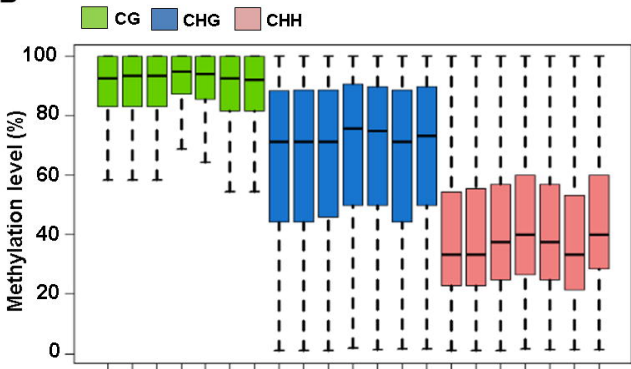

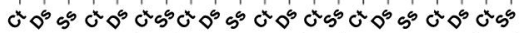

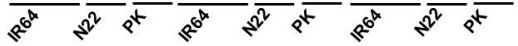

C

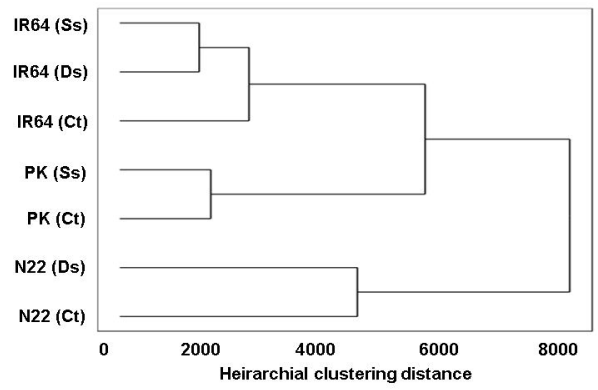

chr1
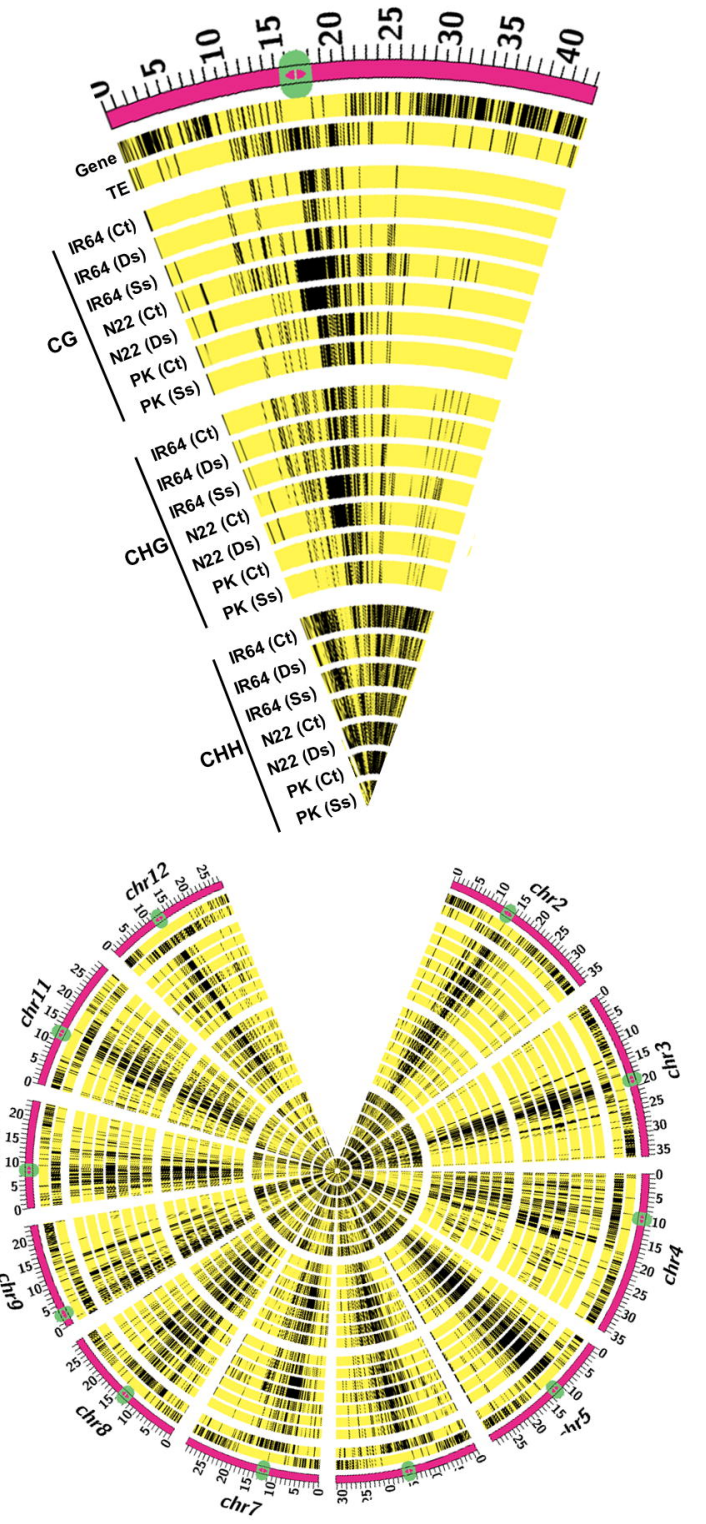

low

high 
A

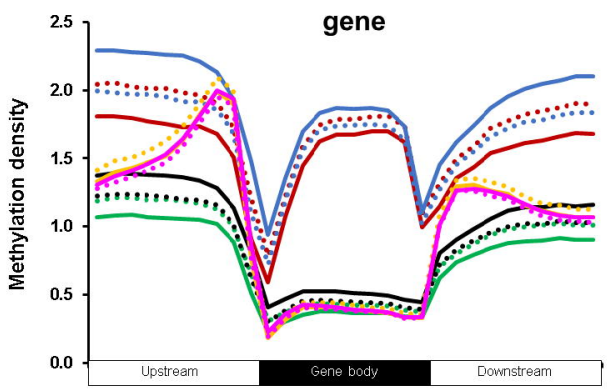

- IR64 CG (Ct) IR64 CHG (Ct) $=\operatorname{IR64} \mathrm{CHH}(\mathrm{Ct})$

". " - |R64 CG (Ds) " " " " IR64 CHG (Ds) = " " IR64 CHH (Ds)

- N22 CG (Ct) N22 CHG (Ct) $=\mathrm{N} 22 \mathrm{CHH}(\mathrm{Ct})$

" = " N22 CG (Ds) " " = N22 CHG (Ds) " " " " N22 CHH (Ds)

C

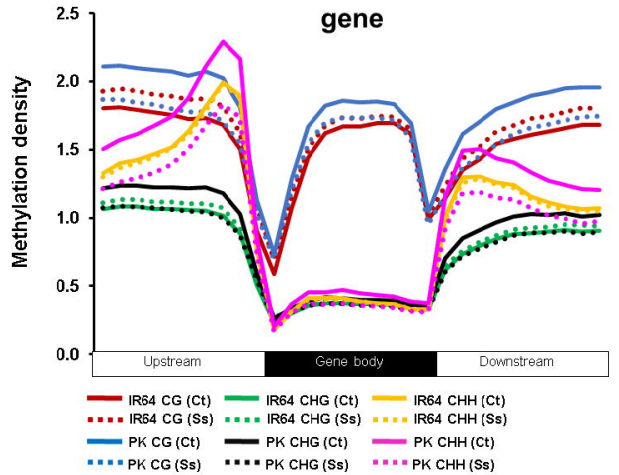

B

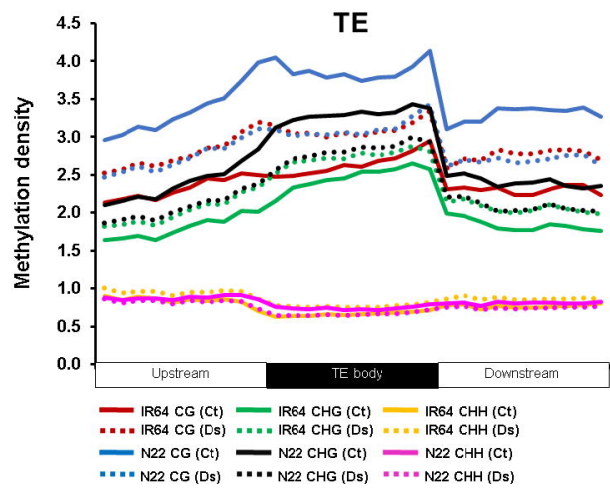

D

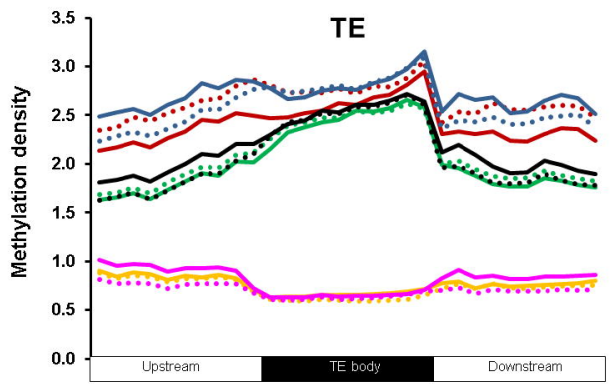

IR64 CG (Ct) IR64 CHG (Ct) $=$ IR64 CHH (Ct)

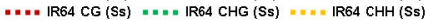

PKCG(Ct) PKCHG (Ct) PKCHH(Ct)

\#-"PKCG(Ss] \#...PKCHG (Ss) \#" " PK CHH(Ss) 
A $\quad$ IR64 (Ct)
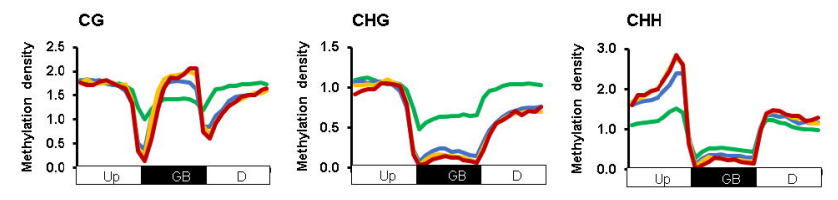

B $\quad$ IR64 (Ds)
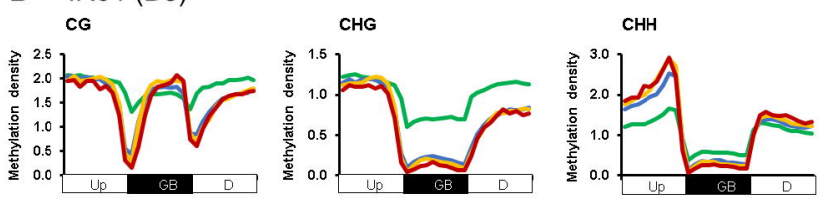

D $\quad \mathrm{N} 22(\mathrm{Ct})$
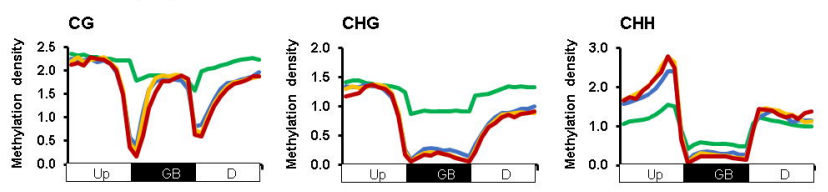

F $\mathrm{PK}(\mathrm{Ct})$
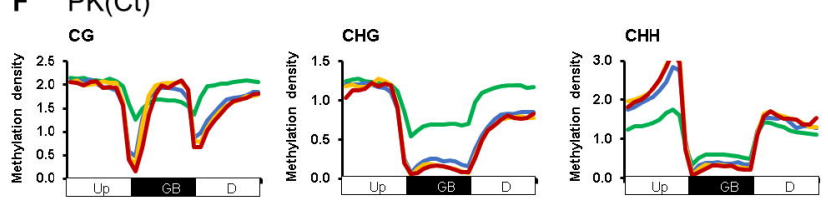

- Silent/very low $(<1)$ low $(\geq 1<5)-$ moderate $(\geq 5<25)-\operatorname{high}(>25)$

C IR64(Ss)
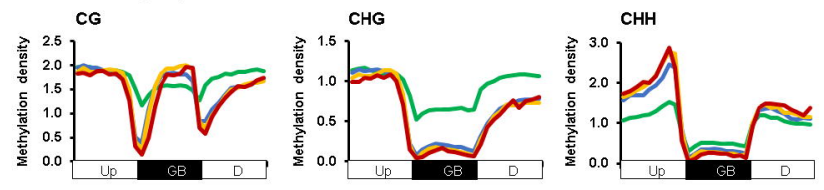

E N22 (Ds)
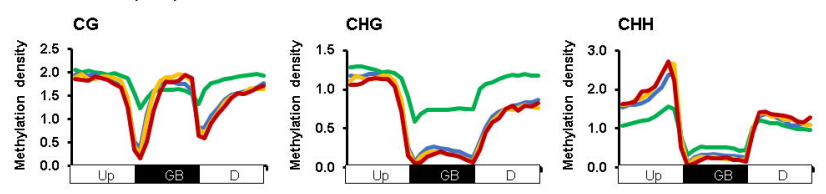

G $\mathrm{PK}(\mathrm{Ss})$
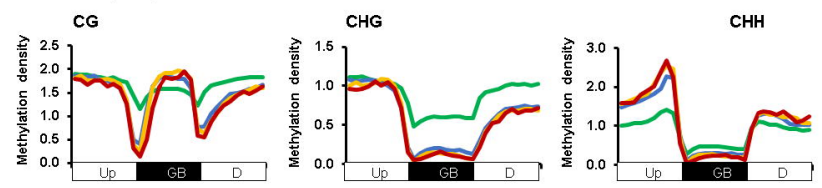


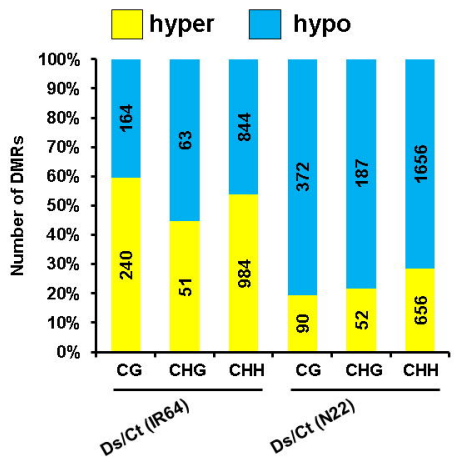

D

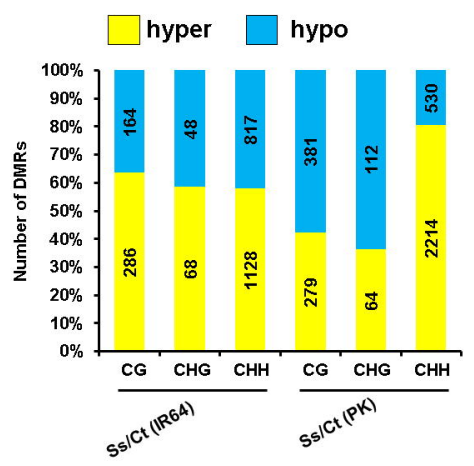

G

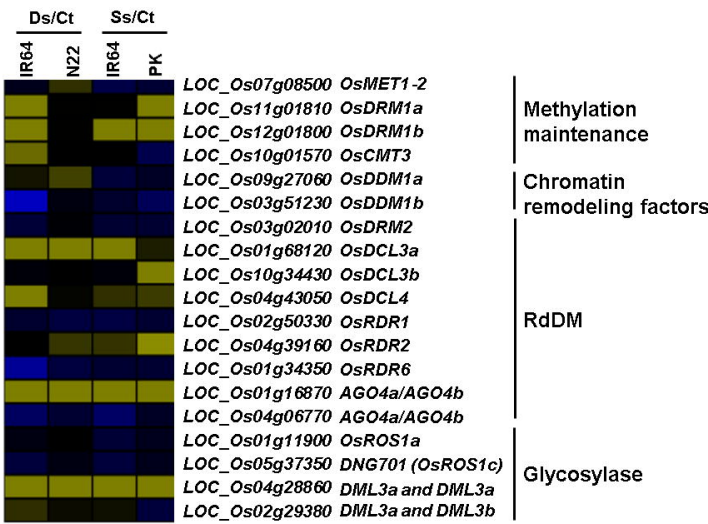

C

IR64 (Ds/Ct)

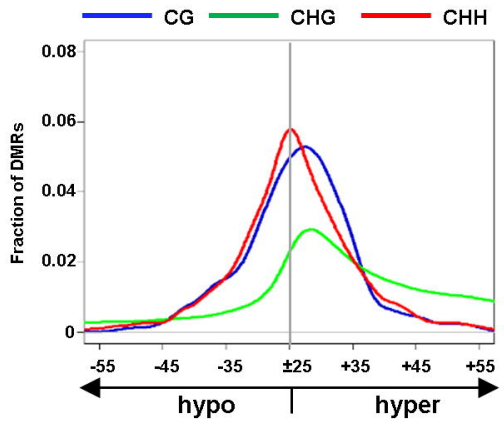

Methylation level difference (\%)

E

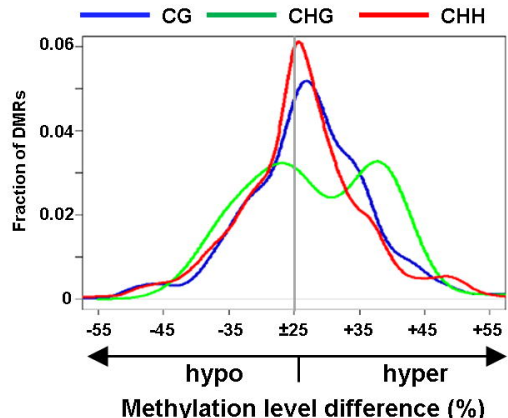

N22 (Ds/Ct)

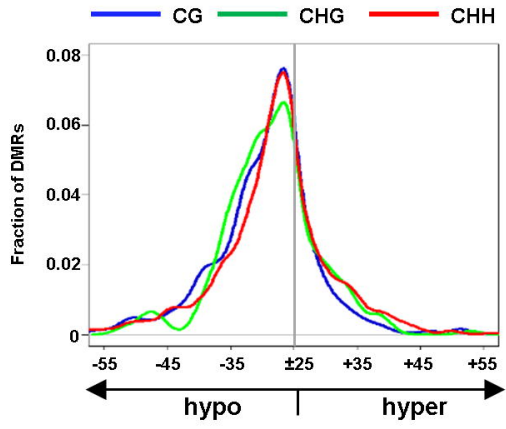

Methylation level difference (\%)

F

PK (Ss/Ct)

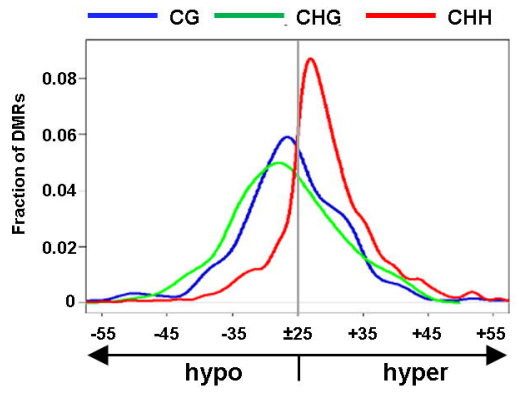

Methylation level difference (\%) 
C

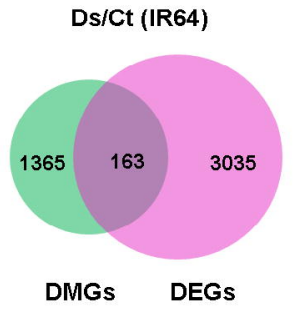

B

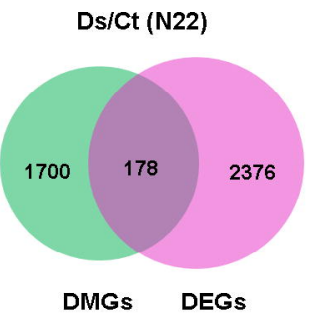

DMGs

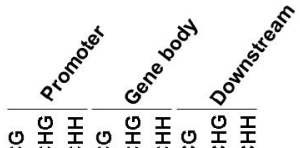
엉 옹 퐁 잉 옹 퐁 잉 옹 퐁

i
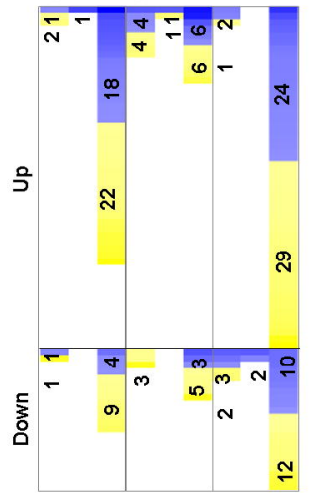

D

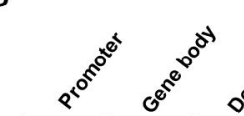

E

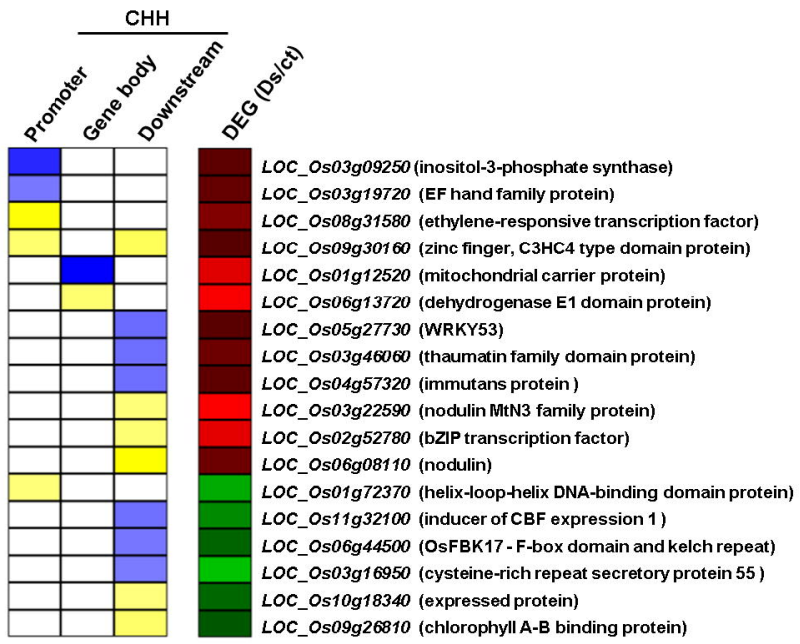

F

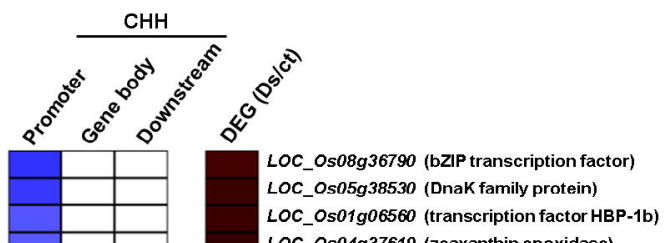

LOC_Os01g06560 (transcription factor HBP-1b)

LOC_Os04g37619 (zeaxanthin epoxidase)

LOC_Os03g15570 (STE_MEKK_ste11_MAP3K.12)

LOC_Os05g27780 (expressed protein)

LOC_Os02g38130 (no apical meristem protein)

LOC_Os05g01460 (STIP1 homology and $U$ box-protein)

LOC_Os01g62900 (amino acid kinase)

LOC_Os08g38400 (zinc finger, C3HC4 type domain)

LOC_Os11g24550 (MBTB61 - Bric-a-Brac, Tramtrack)

LOC_Os04g57340 (AP2 domain containing protein)

LOC_Os01g16430 (cysteine proteinase inhibitor protein)

LOC_Os09g37100 (phospholipase D)

LOC_Os09g26160 (glutamate receptor)

LOC_Os07g42910 (cytochrome c oxidase subunit)

LOC_Os02g56920 (WAX2)

LOC_Os01g22249 (peroxidase precursor)

LOC_Os11g32100 (inducer of CBF expression 1)

LOC_Os12g26940 (CHASE domain containing protein)

LOC_Os10g02480 (oxidoreductase, aldo/keto reductase)

LOC_Os03g62780 (S1 RNA binding domain containing protein)

LOC_Os11g01140 (ACG kinases, homologs to PKA, PKG, PKC) 
A

Ss/Ct (IR64)

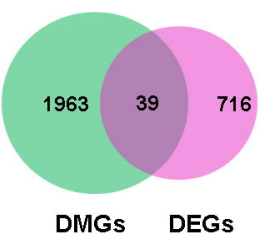

B

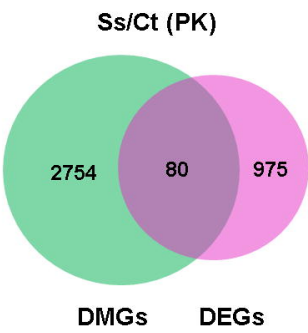

C

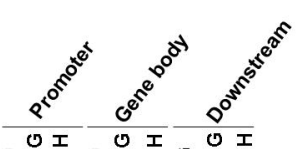

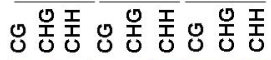
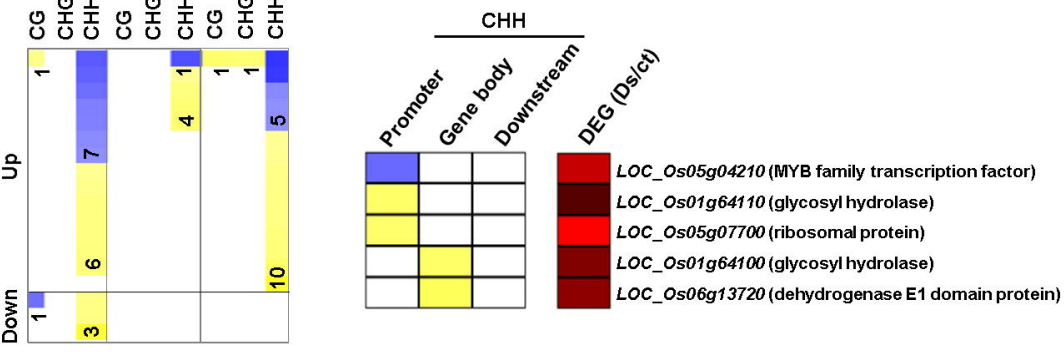

F

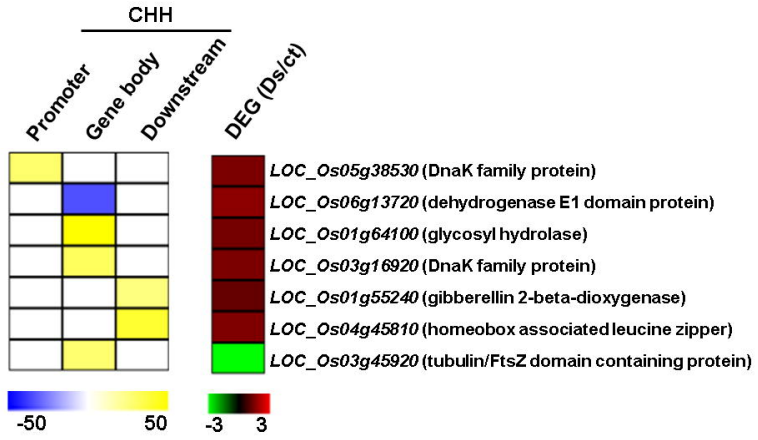

D

s

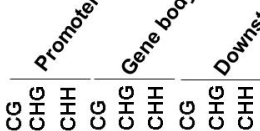

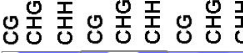

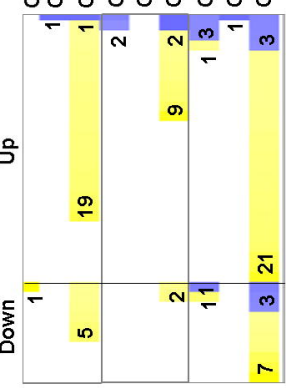

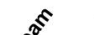

